\title{
MOORE-PENROSE INVERSION IN COMPLEX CONTRACTED INVERSE SEMIGROUP ALGEBRAS
}

\author{
W. D. MUNN
}

(Received 13 November 1998; revised 3 March 1999)

Communicated by D. Easdown

\begin{abstract}
It is shown that every element of the complex contracted semigroup algebra of an inverse semigroup $S=S^{0}$ has a Moore-Penrose inverse, with respect to the natural involution, if and only if $S$ is locally finite. In particular, every element of a complex group algebra has such an inverse if and only if the group is locally finite.
\end{abstract}

1991 Mathematics subject classification (Amer. Math. Soc.): primary 20M25.

Let $A$ be an algebra over the complex field $\mathbb{C}$ with an involution *. By a MoorePenrose inverse of an element $a \in A$ (relative to $*$ ) we mean an element $a^{\dagger} \in A$ such that

$$
\begin{aligned}
& a a^{\dagger} a=a, \quad a^{\dagger} a a^{\dagger}=a^{\dagger}, \\
& \left(a a^{\dagger}\right)^{*}=a a^{\dagger}, \quad\left(a^{\dagger} a\right)^{*}=a^{\dagger} a .
\end{aligned}
$$

It is readily demonstrated that there is at most one such $a^{\dagger}$ for a given $a$ (see [6]); and clearly $0=0^{\dagger}$. The fundamental case is that in which $A$ is the algebra $M_{n}(\mathbb{C})$ of all $n \times n$ matrices over $\mathbb{C}$ and ${ }^{*}$ is hermitian conjugation. In [6, Theorem 1], Penrose proved that $a^{\dagger}$ exists for each $a \in M_{n}(\mathbb{C})$. An equivalent result, using a different definition of $a^{\dagger}$, had been obtained earlier by Moore [2]. The purpose of this note is to extend Penrose's theorem to a wider class of complex algebras.

The semigroup algebra of a semigroup $S$ over $\mathbb{C}$ is designated by $\mathbb{C}[S]$. Adopting the convention in [1], we write ' $S=S^{0}$ ' to indicate that a semigroup $S$ has a zero and at least one other element. Given such a semigroup $S$, we denote the set of nonzero elements of $S$ by $\hat{S}$ and the contracted semigroup algebra of $S$ over $\mathbb{C}$ by $\mathbb{C}_{0}[S]$

(C) 1999 Australian Mathematical Society 0263-6115/99 \$A2.00+0.00 
[1, Section 5.2]. The elements of $\mathbb{C}_{0}[S]$ are regarded as the formal sums $\sum_{x \in \hat{S}} \alpha_{x} x$, where in each case at most finitely many of the (complex) coefficients $\alpha_{x}$ are nonzero. Multiplication in $\mathbb{C}_{0}[S]$ is induced by that in $S$ in the obvious way, the zero of $S$ being identified with the zero of the algebra. For a typical element $a=\sum_{x \in \hat{S}} \alpha_{x} x$ we define $\operatorname{supp}(a)$, the support of $a$, to be $\left\{x \in \hat{S}: \alpha_{x} \neq 0\right\}$. Thus supp $(a)$ is a finite subset of $\hat{S}$ and is empty if and only if $a=0$. (Note that every semigroup algebra can be viewed as a contracted semigroup algebra; for if $T$ is an arbitrary semigroup then $\mathbb{C}[T]=\mathbb{C}_{0}\left[T^{+}\right]$, where $T^{+}$is obtained from $T$ by adjoining a zero.)

A semigroup $S$ is said to be locally finite if and only if every finite nonempty subset of $S$ generates a finite subsemigroup of $S$. The following result, which is a special case of [5, Theorem 2], provides a necessary condition for a complex contracted semigroup algebra to be regular. (An algebra $A$ is regular, in the sense of von Neumann, if and only if, for all $a \in A$ there exists $x \in A$ such that $a x a=a$.)

LEMMA 1 (Okniński). Let $S=S^{0}$ be a semigroup. If $\mathbb{C}_{0}[S]$ is regular then $S$ is locally finite.

We now confine our discussion to inverse semigroups. A semigroup $S$ of this type has the defining property that to each $x \in S$ there corresponds a unique $x^{-1} \in S$ (the 'inverse' of $x$ ) such that $x x^{-1} x=x$ and $x^{-1} x x^{-1}=x^{-1}$. It can be shown that the idempotents of $S$ necessarily commute and that the inversion $\left(x \mapsto x^{-1}\right)$ is an involution on $S$ [1, Theorem 1.17 and Lemma 1.18]. Now consider an inverse semigroup $S=S^{0}$. For each $\alpha \in \mathbb{C}$ denote the complex conjugate of $\alpha$ by $\bar{\alpha}$. Then the mapping ${ }^{*}: \mathbb{C}_{0}[S] \rightarrow \mathbb{C}_{0}[S]$ defined by

$$
\left(\sum_{x \in \hat{S}} \alpha_{x} x\right)^{*}:=\sum_{x \in \hat{S}} \overline{\alpha_{x}} x^{-1} \quad\left(\alpha_{x} \in \mathbb{C}\right)
$$

is readily seen to be an involution. We call this the natural involution on $\mathbb{C}_{0}[S]$. For the case in which $S$ is the semigroup of $n \times n$ matrix units (that is,

$$
\left.S=\left\{e_{i j}: 1 \leq i, j \leq n\right\} \cup\{0\}, \quad \text { with } e_{i j} e_{k l}=\delta_{j k} e_{i l}\right),
$$

$\mathbb{C}[S]=M_{n}(\mathbb{C})$ and ${ }^{*}$ coincides with the hermitian conjugation.

A version of the next lemma, for a non-contracted complex inverse semigroup algebra, was used by the author to show that the algebra has no nonzero nil ideals [4, Lemma 2.3]. With minor adjustment, the proof applies also to the contracted case. The same result was obtained independently by Shehadah [8].

LEMMA 2 (Munn-Shehadah). Let $S=S^{0}$ be an inverse semigroup and let ${ }^{*}$ denote the natural involution on $\mathbb{C}_{0}[S]$. Then

$$
\left(\forall a \in \mathbb{C}_{0}[S]\right) \quad a a^{*}=0 \Rightarrow a=0 .
$$


Before proceeding to the main result we observe the following. Let $S$ be an inverse semigroup, let $T$ be a finite nonempty subset of $S$ and let $T^{-1}$ denote $\left\{x^{-1}: x \in T\right\}$. Then the inverse subsemigroup of $S$ generated by $T$ is the subsemigroup generated by $T \cup T^{-1}$. Thus $S$ is locally finite if and only if every finite nonempty subset of $S$ generates a finite inverse subsemigroup of $S$.

THEOREM 1. Let $S=S^{0}$ be an inverse semigroup. Then every element of $\mathbb{C}_{0}[S]$ has a Moore-Penrose inverse, relative to the natural involution, if and only if $S$ is locally finite.

Proof. Assume first that every element of $\mathbb{C}_{0}[S]$ has a Moore-Penrose inverse. Then, in particular, $\mathbb{C}_{0}[S]$ is regular and so, by Lemma $1, S$ is locally finite.

For the converse part, we adapt Penrose's argument in [6, Theorem 1]. Denote the natural involution on $\mathbb{C}_{0}[S]$ by ${ }^{*}$. We show first that, for all $a, b, c \in \mathbb{C}_{0}[S]$,
(i) $b a^{*} a=c a^{*} a \Rightarrow b a^{*}=c a^{*}$,
(ii) $b a a^{*}=c a a^{*} \Rightarrow b a=c a$.

To see that (i) holds, suppose that $a, b, c \in \mathbb{C}_{0}[S]$ are such that $b a^{*} a=c a^{*} a$. Then $\left(b a^{*}-c a^{*}\right)\left(b a^{*}-c a^{*}\right)^{*}=\left(b a^{*} a-c a^{*} a\right)(b-c)^{*}=0$ and so, by Lemma $2, b a^{*}=c a^{*}$. Result (ii) follows by replacing $a$ by $a^{*}$ in (i).

Now assume that $S$ is locally finite. Consider a nonzero element $a$ of $\mathbb{C}_{0}[S]$. Let $T\left(=T^{0}\right)$ denote the inverse subsemigroup of $S$ generated by $\operatorname{supp}(a) \cup\{0\}$. Then $a a^{*} \in \mathbb{C}_{0}[T]$. But $\mathbb{C}_{0}[T]$ is finite-dimensional. Hence, for some $k \geq 2$, there exist complex numbers $\lambda_{1}, \lambda_{2}, \ldots, \lambda_{k}$, with $\lambda_{i} \neq 0$ for some $i<k$, such that $\sum_{i=1}^{k} \lambda_{i}\left(a a^{*}\right)^{i}=0$. Applying ${ }^{*}$ to both sides, we see that also $\sum_{i=1}^{k} \bar{\lambda}_{i}\left(a a^{*}\right)^{i}=0$. From these equations, it follows readily that there exist real numbers $\mu_{1}, \mu_{2}, \ldots, \mu_{k}$, with $\mu_{i} \neq 0$ for some $i<k$, such that

$$
\mu_{1}\left(a a^{*}\right)+\mu_{2}\left(a a^{*}\right)^{2}+\cdots+\mu_{k}\left(a a^{*}\right)^{k}=0 .
$$

Let $r$ be the smallest integer $i$ for which $\mu_{i} \neq 0$. Define $x \in \mathbb{C}_{0}[S]$ by

$$
x:=-\mu_{r}^{-1}\left[\mu_{r+1} a^{*}+\mu_{r+2} a^{*}\left(a a^{*}\right)+\cdots+\mu_{k} a^{*}\left(a a^{*}\right)^{k-r-1}\right] .
$$

Clearly, $(a x)^{*}=a x$ and $(x a)^{*}=x a$. Further, it is easily verified that $a x\left(a a^{*}\right)^{r}=$ $\left(a a^{*}\right)^{r}$ and so, by repeated applications of (i) and (ii), $a x a a^{*}=a a^{*}$. Therefore $(a x a-a)(a x a-a)^{*}=\left(a x a a^{*}-a a^{*}\right) x^{*} a^{*}-\left(a x a a^{*}-a a^{*}\right)=0$. Hence, by Lemma 2, axa $=a$. From this, we have that $x a x a=x a$; also $a^{*}=a^{*} x^{*} a^{*}$ and so $x=y a^{*}$ for some $y \in \mathbb{C}_{0}[S]$. Thus $x a y a^{*} a=y a^{*} a$. Hence, by (i), xay $a^{*}=y a^{*}$; that is, $x a x=x$. Consequently, $x$ is the Moore-Penrose inverse of $a$ in $\mathbb{C}_{0}[S]$.

REMARKS. (1) Penrose's result on $M_{n}(\mathbb{C})$ is included as a special case of Theorem 1: take $S$ to be the semigroup of $n \times n$ matrix units. 
(2) Note that $\mathbb{C}_{0}[S]$ need not have an identity element. However, for a finite inverse semigroup $T=T^{0}, \mathbb{C}_{0}[T]$ does have an identity. A formula expressing this element in terms of the idempotents of $T$ has been obtained by Penrose (see [3, p. 11]).

We conclude with a discussion of equations of the form $a x=b$ in complex contracted inverse semigroup algebras. First, it is easy to verify (as in [6]) that, if $a$ and $b$ are elements of an arbitrary ring $R$ and there exists $a^{\prime} \in R$ such that $a a^{\prime} a=a$, then the equation $a x=b$ is soluble in $R$ if and only if $a a^{\prime} b=b$, the solutions (where they exist) being precisely the elements of the form $a^{\prime} b+r-a^{\prime} a r$, where $r$ ranges over $R$. In particular, these observations apply when $R$ is a complex contracted inverse semigroup algebra and $a^{\prime}=a^{\dagger}$.

Let $S=S^{0}$ be a semigroup. We define the (Euclidean) norm $\|a\|$ of $a \in \mathbb{C}_{0}[S]$ by the rule that, if $a=\sum_{x \in \hat{S}} \alpha_{x} x$, then

$$
\|a\|:=\left(\sum_{x \in \hat{S}}\left|\alpha_{x}\right|^{2}\right)^{1 / 2} .
$$

Following Penrose [7], for $a$ and $b$ in $\mathbb{C}_{0}[S]$ we say that $t \in \mathbb{C}_{0}[S]$ is a best approximate solution of the equation $a x=b$ if and only if, for all $u \in \mathbb{C}_{0}[S]$, (1) $\|a t-b\| \leq\|a u-b\|$ and (2) if $\|a t-b\|=\|a u-b\|$ then $\|t\| \leq\|u\|$.

Now suppose that $S=S^{0}$ is an inverse semigroup. Denote the set of all nonzero idempotents of $S$ by $\hat{E}$. We say that $S$ is primitive if and only if

$$
(\forall e, f \in \hat{E}) \quad \text { ef } \neq 0 \Rightarrow e=f .
$$

It can be shown [1, Section 6.5, Example 6] that $S$ is primitive if and only if it is adirect union of Brandt semigroups (that is, completely 0 -simple inverse semigroups). Thus complex contracted semigroup algebras of primitive inverse semigroups include, as special cases, complex group algebras and full matrix algebras over $\mathbb{C}$.

The next theorem mirrors Penrose's result on best approximate solutions of matrix equations [7].

THEOREM 2. Let $S=S^{0}$ be a primitive inverse semigroup, let $a$ and $b$ be elements of $\mathbb{C}_{0}[S]$ and assume that $a^{\dagger}$ exists. Then $a^{\dagger} b$ is the unique best approximate solution of $a x=b$ in $\mathbb{C}_{0}[S]$.

REMARK. By Theorem 1, a sufficient condition for $a^{\dagger}$ to exist is that the (inverse) subsemigroup of $S$ generated by supp ( $a$ ) is finite.

Proof. Let $x, y \in \hat{S}$ be such that $x^{-1} y \in \hat{E}$. Then, since $x^{-1} x \in \hat{E}$ and $\left(x^{-1} x\right)\left(x^{-1} y\right) \neq 0$ we have that $x^{-1} y=x^{-1} x$ and therefore that $x^{-1} y x^{-1}=x^{-1}$. 
Similarly, $y x^{-1} y=y$. Hence $y=\left(x^{-1}\right)^{-1}=x$. Thus we have that

$$
(\forall x, y \in \hat{S}) \quad x^{-1} y \in \hat{E} \Leftrightarrow x=y .
$$

Next, we define a linear functional $\tau: \mathbb{C}_{0}[S] \rightarrow \mathbb{C}$ by the rule that

$$
\tau\left(\sum_{x \in \hat{S}} \alpha_{x} x\right):=\sum_{e \in \hat{E}} \alpha_{e} \quad\left(\alpha_{x} \in \mathbb{C}\right)
$$

As before, denote the natural involution on $\mathbb{C}_{0}[S]$ by ${ }^{*}$. It follows readily from (1) that

$$
\left(\forall u \in \mathbb{C}_{0}[S]\right) \quad \tau\left(u^{*} u\right)=\|u\|^{2} .
$$

Let $u \in \mathbb{C}_{0}[S]$ and write $c:=a u-a a^{\dagger} b, \quad d:=a a^{\dagger} b-b$. Then

$$
c^{*} d=\left(u^{*}-\left(a^{\dagger} b\right)^{*}\right) a^{*}\left(a a^{\dagger} b-b\right)=0,
$$

since $a^{*}\left(a a^{\dagger}\right)=\left(a a^{\dagger} a\right)^{*}=a^{*}$. Thus, by (2) and (3),

$$
\begin{aligned}
\|a u-b\|^{2}=\|c+d\|^{2} & =\tau\left((c+d)^{*}(c+d)\right)=\tau\left(c^{*} c+c^{*} d+\left(c^{*} d\right)^{*}+d^{*} d\right) \\
& =\tau\left(c^{*} c+d^{*} d\right)=\tau\left(c^{*} c\right)+\tau\left(d^{*} d\right)=\|c\|^{2}+\|d\|^{2}
\end{aligned}
$$

Hence $\|d\| \leq\|a u-b\|$; that is, $\left\|a a^{\dagger} b-b\right\| \leq\|a u-b\|$.

Suppose now that equality holds here. Then $\|c\|=0$ and so $a u=a a^{\dagger} b$. Thus $a^{\dagger} a u=a^{\dagger} b$. Consequently, $u=e+f$, where $e:=a^{\dagger} b$ and $f:=u-a^{\dagger} a u$. Now $\left(a^{\dagger}\right)^{*}\left(u-a^{\dagger} a u\right)=0$ and so $e^{*} f=0$. Hence, by (2),

$$
\|u\|^{2}=\tau\left((e+f)^{*}(e+f)\right)=\tau\left(e^{*} e+f^{*} f\right)=\|e\|^{2}+\|f\|^{2} .
$$

Therefore $\|e\| \leq\|u\| ;$ that is, $\left\|a^{\dagger} b\right\| \leq\|u\|$.

Finally, suppose additionally that $\left\|a^{\dagger} b\right\|=\|u\|$. Then $\|f\|=0$. Hence $f=0$ and so $u=e=a^{\dagger} b$. Thus we have shown that $a^{\dagger} b$ is the unique best approximate solution of $a x=b$ in $\mathbb{C}_{0}[S]$.

\section{Acknowledgments}

I am grateful to Dr M. J. Crabb and Dr D. Easdown for some valuable advice on the preparation of this note. 


\section{References}

[1] A. H. Clifford and G. B. Preston, The algebraic theory of semigroups, Math. Surveys of the Amer. Math. Soc. 7 (Providence R.I., 1961 (vol. I) and 1967 (vol. II)).

[2] E. H. Moore, 'On the reciprocal of the general algebraic matrix', Bull. Amer. Math. Soc. 26 (1920), 394-395.

[3] W. D. Munn, 'Matrix representations of semigroups', Proc. Cambridge Philos. Soc. 53 (1957), 5-12.

[4] - 'Semiprimitivity of inverse semigroup algebras', Proc. Roy. Soc. Edinburgh, Sect. A 93 (1982), 83-98.

[5] J. Okniński, 'On regular semigroup rings', Proc. Roy. Soc. Edinburgh, Sect. A 99 (1984), 145-151.

[6] R. Penrose, 'A generalized inverse for matrices', Proc. Cambridge Philos. Soc. 51 (1955), 406-413.

[7] _-, 'On best approximate solutions of linear matrix equations', Proc. Cambridge Philos. Soc. 52 (1956), 17-19.

[8] A. A. Shehadah, Embedding theorems for semigroups with involution (Ph.D. Thesis, Purdue University, Indiana, 1982).

Department of Mathematics

University of Glasgow

Glasgow G12 8QW

Scotland

UK

e-mail: wdm@maths.gla.ac.uk 